she did not have any neurological deficit. Investigations including EEG and CT scan of the head did not reveal any abnormality.

Some believe that impaired visual acuity and a disorder of brain function are both required for CBS to develop but it is also known to develop in individuals with normal vision (Podoll et al, 1989). There is no consensus on whether pathology in the visual system is necessary, possible or incompatible with the diagnosis of CBS (Hecaen \& Albert, 1975).

In this patient, complex, isolated, persistent and recurrent visual hallucinations were hypnogogic in nature. Lesions of diencephalon and also diffuse lesions of cortex can produce hypnogogic hallucinations which are predominantly or solely visual, and the aetiology of CBS may lie in structural or functional abnormalities of this region.

HeCAen, H. \& Albert, M. L. (1975) Human Neuropsychology. New York: Wiley.

PODOLL, K., OSTERHEIDER, M. \& NoTH, J. (1989) Das CharlesBonnet-Syndrom. Fortschritte Der Neurologie-Psychiatrie, 57, 43-60.

Teunisse, R. J., Cruysberg, J. R. M., Verbeek, A., et al (1995) The Charles Bonnet Syndrome: A large prospective study in the Netherlands. British Journal of Psychiatry, 166, 254-257.

Masterton Hospital

D.K. ARYa

P.O. Box 96, Masterton

New Zealand

SIR: We commend Teunisse et al (BJP, February $1995,166,254-257)$ for the first ever major case finding study using fixed criteria and a control group. However the authors' assertion that in other prevalence studies on complex visual hallucinations in visually impaired patients, the concept of Charles Bonnet syndrome (CBS) was not used is somewhat unjustified.

There is considerable confusion with the use of the term CBS by both psychiatrists and ophthalmologists. Originally defined as visual hallucinations in elderly patients without evidence of ophthalmological impairment, the term has been modified over the years to include visual hallucinatory phenomena of a pleasant or neutral nature in conjunction with a clear state of consciousness (Damas-Mora et al, 1982). Within this modification is the fact that it is often associated with ocular disease. There seems to be no consensus however, about the relationship between eye pathology, brain lesions and CBS. At least two definitions exist (Damas-Mora et al, 1982; Gold \& Rabins, 1989). The authors criteria in the study are almost identical to the criteria proposed by Gold \& Rabins.
Two recent prevalence studies not mentioned by the authors are worthy of mention. Norton-Willson \& Munir (1987), in a retrospective study of 434 consecutive patients referred for consultation to a psychogeriatric unit over a period of 3.5 years, reported eight cases of visual perceptual disorders resembling CBS. Brown \& Murphy (1992) studied 100 consecutive patients with macular choroidal neo-vascularisation in a cross-sectional fashion and reported 12 subjects with formed hallucinations (Charles Bonnet syndrome). These authors have not defined or used any criteria for CBS preferring instead to use the term visual perceptual disorders or formed visual hallucinations.

We suggest that if systematic research into this interesting phenomenon should meaningfully progress, some consensus on the criteria for CBS should be arrived at, or else we risk having to give up the use of this eponym which has so far stood the test of time.

Brown, G. C. \& MurPhy, R. P. (1992) Visual symptoms associated with choroidal neovascularisation. Photopsias and the Charles Bonnet syndrome. Archives of Ophthalmology, 110, 1251-1256.

Damas-Mora, J., Skelton-Robinson, M. \& Jenner, F. A. (1982)

The Charles Bonnet syndrome in perspective. Psychological Medicine, 12, 251-261.

GolD, K. \& RABINS, P. V. (1989) Isolated visual hallucinations and the Charles Bonnet syndrome: a review of the literature and presentation of six cases. Comprehensive Psychiatry, 30, 90-98.

NORTON-WILLSON, L. \& MUNIR, M. (1987) Visual perceptual disorders resembling the Charles Bonnet syndrome. A study of 434 consecutive patients referred to a psychogeriatric unit. Family Practice, 4, 27-35.

A. FERNANDEZ

G. LICHTSHEIN

Medical College of Virginia

P.O. Box 1326

Richmond, VA 23298-1326

\section{The cultural context of hallucinations}

SIR: Al-Issa (1995) is right to emphasise the importance of the cultural context of hallucinations. But his discussion of the origins of hallucinations in terms of cultural attitudes appears incomplete.

Al-Issa distinguishes "rational" cultures, which make a rigid distinction between reality and fantasy, from the "less rational" cultures which have a more flexible distinction. In his view, a rigid distinction promotes negative attitudes towards hallucinations and makes people less introspective, less familiar with the workings of their own imagination, and so less aware of imaginings such as hallucinations. 